Subscriber access provided by King Abdullah University of Science and Technology Library

\title{
Article
}

\section{Selectively Plasmon-Enhanced Second-Harmonic Generation from Monolayer Tungsten Diselenide on Flexible Substrates}

Zhuo Wang, Zhaogang Dong, Hai Zhu, Lei Jin, Ming-Hui Chiu, Lain-Jong Li, Qing-Hua

Xu, Goki Eda, Stefan A. Maier, Andrew T. S. Wee, Cheng-Wei Qiu, and Joel K.W. Yang

ACS Nano, Just Accepted Manuscript • DOI: 10.1021/acsnano.7b08682 • Publication Date (Web): 04 Jan 2018

Downloaded from http://pubs.acs.org on January 17, 2018

\section{Just Accepted}

"Just Accepted" manuscripts have been peer-reviewed and accepted for publication. They are posted online prior to technical editing, formatting for publication and author proofing. The American Chemical Society provides "Just Accepted" as a free service to the research community to expedite the dissemination of scientific material as soon as possible after acceptance. "Just Accepted" manuscripts appear in full in PDF format accompanied by an HTML abstract. "Just Accepted" manuscripts have been fully peer reviewed, but should not be considered the official version of record. They are accessible to all readers and citable by the Digital Object Identifier (DOI®). "Just Accepted" is an optional service offered to authors. Therefore, the "Just Accepted" Web site may not include all articles that will be published in the journal. After a manuscript is technically edited and formatted, it will be removed from the "Just Accepted" Web site and published as an ASAP article. Note that technical editing may introduce minor changes to the manuscript text and/or graphics which could affect content, and all legal disclaimers and ethical guidelines that apply to the journal pertain. ACS cannot be held responsible for errors or consequences arising from the use of information contained in these "Just Accepted" manuscripts. 


\section{Selectively Plasmon-Enhanced Second-Harmonic Generation from Monolayer Tungsten Diselenide on Flexible Substrates}

Zhuo Wang ${ }^{1,2,3, \perp}$, Zhaogang Dong ${ }^{4, \perp}$, Hai Zhu ${ }^{5, \perp}$, Lei Jin ${ }^{6}$, Ming-Hui Chiu ${ }^{7}$, Lain-Jong Li $^{7}$, Qing-Hua $\mathrm{Xu}^{5}$, Goki Eda ${ }^{2,5,8}$, Stefan A. Maier ${ }^{3}$, Andrew T. S. Wee ${ }^{1,2,8 *}$, Cheng-Wei Qiu ${ }^{1,6,8 *}$, Joel K. W. Yang ${ }^{4,9, *}$

${ }^{1}$ NUS Graduate School for Integrative Sciences \& Engineering (NGS), National University of Singapore, 28 Medical Drive, Singapore117456, Singapore

${ }^{2}$ Department of Physics, National University of Singapore, 2 Science Drive 3, Singapore 117542 , Singapore

${ }^{3}$ Department of Physics, Imperial College London, London SW7 2AZ, UK

${ }^{4}$ Institute of Materials Research and Engineering, A*STAR (Agency for Science, Technology and Research), 2 Fusionopolis Way, \#08-03 Innovis, Singapore 138634, Singapore

${ }^{5}$ Department of Chemistry, National University of Singapore, 3 Science Drive 3, Singapore 117543, Singapore

${ }^{6}$ Department of Electrical and Computer Engineering, National University of Singapore, 4 Engineering Drive 3, Singapore 117583, Singapore

${ }^{7}$ Physical Science and Engineering Division, King Abdullah University of Science and Technology, Thuwal 23955-6900, Kingdom of Saudi Arabia

${ }^{8}$ Centre for Advanced 2D Materials, National University of Singapore, 2 Science Drive 3, Singapore 117542

${ }^{9}$ Singapore University of Technology and Design, 8 Somapah Road, Singapore 487372, Singapore

\footnotetext{
${ }^{\perp}$ The authors make equal contribution

*Corresponding authors:

Joel K. W. Yang (email: joel_yang@sutd.edu.sg)

Cheng-Wei Qiu (email: chengwei.qiu@nus.edu.sg)

Andrew T. S. Wee (email: phyweets@nus.edu.sg)
} 


\begin{abstract}
Monolayer two-dimensional transition metal dichalcogenides (2D TMDCs) exhibit promising characteristics in miniaturized nonlinear optical frequency converters, due to their inversion asymmetry and large second-order nonlinear susceptibility. However, these materials usually have a very short light interaction lengths with the pump laser because they are atomically thin, such that second-harmonic generation $(\mathrm{SHG})$ is generally inefficient. In this paper, we fabricate a judiciously structured 150-nm-thick planar surface consisting of monolayer tungsten diselenide and sub-20-nm-wide gold trenches on flexible substrates, reporting $\sim 7000$-fold SHG enhancement without peak broadening or background in the spectra as compared to $\mathrm{WSe}_{2}$ on asgrown sapphire substrates. Our proof-of-concept experiment yields effective second-order nonlinear susceptibility of $2.1 \times 10^{4} \mathrm{pm} / \mathrm{V}$. Three orders of magnitude enhancement is maintained with pump wavelength ranging from $800 \mathrm{~nm}$ to $900 \mathrm{~nm}$, breaking the limitation of narrow pump wavelength range for cavity-enhanced SHG. In addition, SHG amplitude can be dynamically controlled via selective excitation of the lateral gap plasmon by rotating the laser polarization. Such fully open, flat and ultrathin profile enables a great variety of functional samples with high SHG from one patterned silicon substrate, favoring scalable production of nonlinear converters. The surface accessibility also enables integration with other optical components for information processing in an ultrathin and flexible form.
\end{abstract}

\footnotetext{
KEYWORDS: Two dimensional (2D) materials, second-harmonic generation, gap plasmon, transition metal dichalcogenides, tungsten diselenide ( $\left.\mathrm{WSe}_{2}\right)$, sub-20-nm nanostructures
} 
Second-harmonic generation (SHG) is the lowest-order frequency mixing nonlinear optical process, where two photons are combined and converted to a new photon with twice the frequency of the input photons. ${ }^{1}$ SHG plays an important role in frequency up-conversion in lasers and optoelectronic hybrid devices. ${ }^{2-7}$ As newly emerging nonlinear materials, twodimensional transition metal dichalcogenides (2D TMDCs), ${ }^{8}$ especially tungsten diselenide $\left(\mathrm{WSe}_{2}\right){ }^{9}$ have attracted tremendous interest as they possess broken inversion-symmetry and relatively large second-order nonlinear susceptibility $\left(\chi^{(2)}\right)$ despite being one monolayer thick. ${ }^{10-}$ ${ }^{12} \chi^{(2)}$ for monolayer TMDCs ranges from 5 to $500 \mathrm{pm} / \mathrm{V}$ depending on the excitation wavelength $^{13}$ and sample quality. ${ }^{2}$ Thus, developing 2D TMDCs as a nonlinear optics source fulfills the demand for smaller and thinner nonlinear optical and optoelectronic devices. ${ }^{14-17}$ However, being atomically thin, 2D TMDCs also possess short light-matter interaction lengths, limiting the total SHG conversion efficiency.

In literature, there are preliminary reports of SHG enhancement in 2D TMDCs. For example, exciton-enhanced SHG, i.e., the enhancement of SHG efficiency at the exciton resonance energy, has been demonstrated. ${ }^{11,18-21}$ At cryogenic temperatures, SHG intensity from monolayer $\mathrm{WSe}_{2}$ can be tuned by changing the wavelength of the pump laser, and it is enhanced by three orders of magnitude when the excitation is two-photon resonant with the exciton energy. ${ }^{18} \mathrm{Nano}^{22}$ or micro-cavities ${ }^{23-24}$ have been demonstrated to enhance SHG in TMDCs at room temperature with enhancements varying from tens to thousands. The enhancement is attributed to the increased electromagnetic wave density achieved by aligning the pump wavelength with the cavity mode ${ }^{22,25}$ However, these cavities made of semiconducting or dielectric materials are not suitable for ultrathin and flexible devices. Nevertheless, by fabricating subwavelength nanostructures patterned on flat surfaces, optical elements can be "flattened" into a 2D sheet of 
less than one wavelength thickness. ${ }^{26-29}$ In the context of developing planar and ultrathin optical devices, there is a compelling case to use ultrathin nanostructures to enhance the SHG of 2D TMDCs.

In this paper, we present a $\mathrm{WSe}_{2}$-gold nanostructure substrate consisting of a subwavelength structure in the form of sub-20-nm-wide trenches on a 150-nm-thick gold film, upon which monolayer $\mathrm{WSe}_{2}$ is transferred. This $\mathrm{WSe}_{2}$-gold trench sample exhibits a significantly larger SHG signal at room temperature with an enhancement factor of $\sim 7000$, as compared to bare $\mathrm{WSe}_{2}$ on sapphire substrates. This value is among the highest SHG enhancement factors for TMDCs reported thus far, corresponding to an effective second-order nonlinear susceptibility $\chi^{(2)}$ of $2.1 \times 10^{4} \mathrm{pm} / \mathrm{V}$. The enhancement is attributed to the enhanced localized near-field of the pump laser at the sub-20-nm-wide trenches, boosting the SHG from the monolayer $\mathrm{WSe}_{2}$. Furthermore, this design allows for easy tuning of the total SHG intensity by rotating the linear polarization of the pump laser. Regardless of the pump laser polarization, the SHG spectra maintain the sharp peak profile of the pump laser. This hybrid nanostructure of monolayer $\mathrm{WSe}_{2}$ on gold trenches is flat and mechanically flexible, with potential for further development of on-chip strain-induced tuning of bendable photonic devices. 


\section{RESULTS AND DISCUSSION}

Flexible nonlinear devices based on hybrid nanostructure of WSe $\mathbf{W}_{2}$-gold trenches. Inversion symmetry breaking ${ }^{30}$ in the crystal structure of monolayer $2 \mathrm{H}$-type $\mathrm{WSe}_{2}$ brings about nonzero $\chi^{(2)}$, enabling $\mathrm{WSe}_{2}$ to support SHG. Hybrid nanostructure, which consists of monolayer $\mathrm{WSe}_{2}$ on a thin gold substrate patterned with sub-20-nm-wide trenches, was prepared to achieve high SHG (Figure 1a). The gold substrate with thickness of $150 \mathrm{~nm}$ was prepared by the templatestripping method. ${ }^{6}$, The monolayer $\mathrm{WSe}_{2}$ flakes were grown on sapphire substrates by a chemical vapor deposition (CVD) method and then immediately transferred onto a freshly prepared gold substrate with sub-20-nm-width trenches by a wet transfer technique (see details in Methods section). The quality and layer number of $\mathrm{WSe}_{2}$ were determined by using optical microscopy (OM), Raman and photoluminescence before transfer (see details in Figure S1). The trenches used here are all aligned along one orientation (Figure S2a) such that the pump laser with electric field polarized perpendicularly to this orientation will excite gap plasmons within the trenches. Conversely, no plasmon excitation is expected when the pump laser is parallelly polarized. Therefore, tuning the polarization direction of the pump laser can switch ON or OFF the gap plasmons in the trenches, thus tuning the SHG intensity of $\mathrm{WSe}_{2}$.

The SHG measurement was conducted by using a femtosecond laser beam generated from a Ti-sapphire oscillator (Coherent, Chameleon Ultra II ) with a central wavelength of 800 $\mathrm{nm}$, pulse duration of $140 \mathrm{fs}$ and repetition rate of $80 \mathrm{MHz}$. The experiment of $\mathrm{SHG}$ as a function of pump wavelengths in Figure S10 used this laser with a central wavelength of $800,825,850$, 875 and $900 \mathrm{~nm}$, respectively. The sample was normally illuminated by the pump laser via a $100 \times$ microscope objective lens with a numerical aperture (NA) of 0.90 (Nikon, Ti-U inverted 
microscope, TU Plan Fluor) and raster scanned by a piezo-actuated 3D nanopositioning stage. At each pixel, the backscattered SHG signal was collected through the same objective and sent to either a photomultiplier tube (PMT) (PicoQuant, PMA 182) or a spectrometer (Princeton Instruments, 2300i). More details about the optical setup can be seen in Methods, Figure S3 and Supplementary Note 1 . Only in the experiments to investigate the orientations of TMDC crystal and trenches, an analyzer was placed before the detectors. Otherwise, the total SHG intensity was not influenced by the crystal orientation of the monolayer $\mathrm{WSe}_{2}$. The SHG intensity was compared by taking peak values at $400 \mathrm{~nm}$ recorded by both PMT and spectrometer. Monolayer $\mathrm{WSe}_{2}$ over trenches, has SHG enhanced by up to $\sim 7000$-fold as compared with $\mathrm{WSe}_{2}$ on sapphire. Unlike previously reported nanocavities or nanostructures, ${ }^{22-23,} 31$ multiple gold nanostructures can be produced from one patterned silicon substrate, favoring scalable production. ${ }^{6}$ Moreover, the 150 -nm-thick hybrid nanostructures provide a flat and flexible top surface, and it serves as an important nonlinear converter for realizing ultrathin and bendable optical information processors on chip. To test the feasibility, the hybrid nanostructure of $\mathrm{WSe}_{2}$ on gold substrate, which was weakly attached to a glass slide via optical adhesive glue, was peeled off from the glass slide using tweezers and transferred to a polydimethylsiloxane (PDMS) substrate of $1 \mathrm{~mm}$ thickness (see fabrication process in Figure S2). The macro shots, scanning electron micrographs (SEM) and SHG intensity of the sample were carefully compared before and after bending. The photo of the unbent sample clearly shows regions with patterned nanostructures on gold film (see Figure 1a). Owing to its nanoscale thickness $(\sim 150 \mathrm{~nm})$ and intrinsic flexibility, the hybrid nanostructure can be bent outward (Figure 1b) and inward (Figure S4) repeatedly in a controllable way once attached to the PDMS substrate. As PDMS substrate was bent outward $\sim 40$ degree off the horizontal line with a bending radius of $2.8 \mathrm{~mm}$, the 
trenches was expanded along the width direction. As a result, the width of trenches on the bent sample $(\sim 17 \mathrm{~nm})$ is slightly larger than that on the unbent sample $(\sim 15 \mathrm{~nm})$ (see Figure $1 \mathrm{c}, \mathrm{d})$. As shown in Figure 1e and f, compared with the unbent sample, the bend does not dramatically affect the SHG intensity of $\mathrm{WSe}_{2}$ on trenches. It is found that after being bent inward and outward for 50 times, the sample can still maintain $70 \%$ of the SHG intensity as the unbent one (Figure S5). For simplicity in handling the samples, the characterization below was conducted on flat samples adhered to glass substrates.

Giant SHG enhancement. To experimentally demonstrate that the SHG from the trenches is significantly enhanced, we transferred a monolayer single-crystalline triangular $\mathrm{WSe}_{2}$ flake on gold trenches spaced at a large pitch of $910 \mathrm{~nm}$. This large pitch of $910 \mathrm{~nm}$ allows us to have the clear mapping of the SHG intensity from the sub-20-nm-wide trench region, based on the farfield optical characterization setup (see Figure S3). Figure 2a shows the SEM image of a triangular single-crystalline $\mathrm{WSe}_{2}$ flake covering 6 trenches. Figure $2 \mathrm{~b}$ presents the simulated electric field distributions over one of these trenches with pump polarization perpendicular (left panel) and parallel (right panel) to the trench. Corresponding to the SEM image, there are 6 brighter SHG stripes in the SHG mapping (Figure 2c) when the polarization direction of incident light is perpendicular to the trench orientation, showing that the SHG enhancement position is localized within the trenches that support lateral gap plasmons. This observation can be corroborated by the simulated localized electric field distribution over the trenches with the pitch of $910 \mathrm{~nm}$ (left panel in Figure 2b). As expected, when the incident light is rotated by 90 degrees, SHG signal from the trenches significantly decreases (Figure 2d), i.e., trenches without the 
excitation of lateral gap plasmons (right panel in Figure 2b) have little influence on SHG from $\mathrm{WSe}_{2}$.

To achieve giant SHG enhancement, we optimized the pitch sizes of the gold nanostructures. The giant SHG intensity was achieved from $\mathrm{WSe}_{2}$ suspended over the trench with a pitch of $170 \mathrm{~nm}$. At the 170-nm pitch size, the lateral gap plasmon resonance is aligned with pump laser wavelength of $800 \mathrm{~nm}$. Figure 3a presents the SEM image of the sample, where a monolayer $\mathrm{WSe}_{2}$ flake is covering both the trench (dark grey) and flat gold film (grey) regions. Figure $3 \mathrm{~b}$ presents the reflectance spectra of the gold trenches ( $\mathrm{T}$ and $\mathrm{T}$ ') and flat gold film (F and $\mathrm{F}^{\prime}$ ) respectively, for both $x$ and $y$ polarizations (see details in Methods section for the reflectance spectrum measurement). The reflectance spectra of the flat gold film (F and F') show no polarization dependence (black and orange lines) as expected due to the isotropic optical properties of gold in the $x-y$ plane. In comparison, the polarization of incident light can turn ON or OFF the lateral gap plasmons in the trenches. Specifically, $x$-polarized incident light excites the lateral gap plasmon resonance seen as the broad absorption dip at $\sim 800 \mathrm{~nm}$ in the reflectance spectrum ( $\mathrm{T}$, red line). In contrast, $y$-polarized incident light with electric field of being polarized along the trench direction cannot excite gap plasmons and exhibit a similar reflectance spectrum as the flat film ( $\mathrm{T}$ ', blue line). The former polarization case is referred to as "resonant excitation" with capital letter representing each sample ( $\mathrm{T}$ for trenches, $\mathrm{F}$ for flat gold or $\mathrm{S}$ for sapphire) and the latter "non-resonant excitation" with apostrophe (T', F' or S') for simplicity. The dips at 400 and $\sim 470 \mathrm{~nm}$ in all reflectance spectra are attributed to the absorption in gold caused by inter-band transitions. $^{32}$

The uniformity of the $\mathrm{WSe}_{2}$ flake is reflected in the uniform intensities in the PL (Figure 3c) and Raman mappings (Figure 3d). In addition, its monolayer nature is confirmed by the 
related spectra (Figure S1e-f), where the PL peak at $\sim 750 \mathrm{~nm}$ and Raman spectrum of two dominant peaks at $\sim 250 \mathrm{~cm}^{-1}$ are indicative of monolayer $\mathrm{WSe}_{2}$. The lateral gap plasmon plays a significant role in enhancing SHG conversion efficiency. Figure $3 \mathrm{e}$ and $3 \mathrm{f}$ show the SHG mappings of $\mathrm{WSe}_{2}$ on gold trenches and flat gold film. The 800-nm pump laser was incident normally to the surface and polarized along $x$-axis (Figure 3e) and $y$-axis (Figure 3f), respectively. As the SHG signal was collected without any analyzer in front of the detector, the total SHG intensity was independent of the crystal orientation of $\mathrm{WSe}_{2}$ with respect to the polarization direction of pump laser. Furthermore, the trenches without $\mathrm{WSe}_{2}$ as shown in the SEM image (Figure S6a) show no observable SHG signal (Figure S6b), indicating that the measured SHG signal is only from the SHG-active material $\mathrm{WSe}_{2}$. Furthermore, the surface nonlinearity of flat gold was insignificant. ${ }^{6,33}$

The as-grown $\mathrm{WSe}_{2}$ on sapphire was taken as the reference sample. As shown in the SHG mapping (Figure S1d), monolayer $\mathrm{WSe}_{2}$ on sapphire has a uniform SHG intensity distribution over the flake. For a quantitative comparison, $4 \mathrm{SHG}$ spectra from $\mathrm{WSe}_{2}$ on the gold trenches (T or T') and sapphire ( $\mathrm{S}$ or S') under $x$ - or $y$-polarized excitation conditions are shown in Figure 4a. Under resonant excitation, the SHG peak value from monolayer $\mathrm{WSe}_{2}$ flake on trenches (T) is enhanced by $\sim 900$-fold on average relative to the reference sample (S). As observed in both simulation and experiments in Figure 2, that SHG enhancement of the sample originates from the trenches. Accounting for the small area fraction (12.6\%) of trenches within the laser spot, the maximum SHG enhancement of $\mathrm{WSe}_{2}$ over the trenches is calculated to be $\sim 7000$-fold. Details of the normalization method are discussed in Figure S7 and Supplementary Note 2. The enhancement arises from the lateral gap plasmon with concentrated electric field in the plane of 
$\mathrm{WSe}_{2}$, increasing the light-matter interaction and enabling the $\mathrm{WSe}_{2}$ flake to more effectively convert 800-nm light into 400-nm light.

Rotating the polarization of pump laser will dynamically tune the total SHG intensity of $\mathrm{WSe}_{2}$ on gold trenches with an extinction ratio of $\sim 47$ (top panel, Figure $4 \mathrm{a}$ ). At the non-resonant excitation condition, the SHG intensity ( $\mathrm{T}^{\prime}$ ) drops to $\sim 1 / 47$ of the counterpart at resonant excitation condition (T) with an enhancement factor of less than 150, owing to the absence of gap plasmons. As expected, no polarization dependence was seen in $\mathrm{WSe}_{2}$ on sapphire (bottom panel, Figure 4a). This conclusion is also verified by the SHG intensity extracted from the SHG mappings (see Figure 4b). Furthermore, it is noted that in both excitation conditions, SHG spectra of $\mathrm{WSe}_{2}$ on gold trenches exhibit a sharp peak at the second-harmonic wavelength (400 $\mathrm{nm}$ ) without spectral broadening or two-photon photoluminescence. The SHG peak is as sharp as the pump laser with a full width and half maximum (FWHM) of $\sim 6 \mathrm{~nm}$, providing a potential light-emission source at shorter wavelength with high monochromaticity (Figure S8). In the application of monochromatic lasing, the sharp peak cannot be achieved by photoluminescence signal that has a broad emission peak $(F W H M=27 \mathrm{~nm})$ (Figure S1e).

SHG enhancement mechanism. For a monolayer $\mathrm{WSe}_{2}$ with $\mathrm{D}_{3 \mathrm{~h}}(\overline{6} m 2)$ point-group symmetry,

34 the second-order nonlinear susceptibility tensor has the following nonzero elements: ${ }^{35}$

$$
\chi_{\mathrm{WSe}_{2}}^{(2)}=\chi_{b b b}^{(2)}=-\chi_{b a a}^{(2)}=-\chi_{a b a}^{(2)}=-\chi_{a a b}^{(2)} \text {, }
$$

where $a$ and $b$ are the principle axes in crystal coordinates. Here, $a$ corresponds to the zigzag direction and $b$ corresponds to the armchair direction that is parallel to a vertical mirror plane (Figure S9). This symmetry leads to a theoretical estimate of the total SHG signal:

$$
I_{2 \omega}=C\left|\mathbf{E}_{2 \omega}\right|^{2} \propto\left|\chi_{b b b}^{(2)}\right|^{2}\left|\mathbf{E}_{\omega}\right|^{4},
$$


where $\mathbf{E}_{2 \omega}$ is the electric field of the second-harmonic frequency wave, $\mathbf{E}_{\omega}$ is the electric field of the pump laser and $C$ is a constant (see eq. S16). The gold trenches are designed to enhance the nonlinear effects of $\mathrm{WSe}_{2}$ by providing near-field enhancement of the pump laser $\left(\mathbf{E}_{\omega}\right)$, at the trenches via the excitation of lateral gap plasmons. Figure $4 \mathrm{c}$ shows the simulated near-field distribution over a trench with a pitch of $170 \mathrm{~nm}$, where the spatial overlap between the enhanced near field of $\mathbf{E}_{\omega}$ and monolayer $\mathrm{WSe}_{2}$ strengthens the light coupling in $\mathrm{WSe}_{2}$ and thus the SH emission. Quantitatively, the near-field of $\mathbf{E}_{\omega}$ is enhanced by $\sim 13$-fold as compared to the electric field of the incident pump laser $\left(\mathbf{E}_{0}\right)$, and thus predicting a theoretical SHG enhancement factor of $13^{4}(\sim 28,000)$. Our SHG enhancement factor, $\sim 7000$, obtained by using $\mathrm{WSe}_{2}$ on sapphire as a reference is only $25 \%$ of the predicted value owing to the partial absorption of the SHG signal at $400 \mathrm{~nm}$ by the gold substrate and partical absorption of the pump caused by the energetic proximity of the exciton in $\mathrm{WSe}_{2}$ with pump. ${ }^{12}$ The reabsorption of the 400 -nm signal can be seen in the reflectance spectrum of gold trenches (Figure 3b). Noted that the trenches has a spectrally broad lateral gap plasmon resonance and extremely narrow mode volume thus having large electric field enhancement for pump with wavelength ranging from $800 \mathrm{~nm}$ to 900 $\mathrm{nm}$. As result, at this wide pump range, the SHG enhancement factor is maintained at thousandsfold for $\mathrm{WSe}_{2}$ on trenches (Figure S10). In addition, our method applies to enhance SHG of various 2D materials such as monolayer $\mathrm{MoS}_{2}$ and $\mathrm{MoSe}_{2}$ with an enhancement factor of thousands-fold (Figure S11).

The effective second-order nonlinear susceptibility $\chi_{\text {eff }}^{(2)}$ was computed using the measured SHG spectra according to the formula: ${ }^{36-38}$

$$
\chi_{e f f}^{(2)} \propto\left(I_{2 \omega}\right)^{1 / 2} / I_{\omega}
$$


where $I_{\omega}$ and $I_{2 \omega}$ are the pump laser and second-harmonic intensities respectively. According to this method, the effective second-order nonlinear susceptibility $\chi_{e f f}^{(2)}$ of $\mathrm{WSe}_{2}$ on gold trenches is calculated to be $\sim 2.1 \times 10^{4} \mathrm{pm} / \mathrm{V}$ with normalization of the trench occupation. In contrast, the effective $\chi_{\text {eff }}^{(2)}$ of our CVD-grown $\mathrm{WSe}_{2}$ on sapphire is $\sim 248 \mathrm{pm} / \mathrm{V}$, which is in the same order as reported values. ${ }^{3,19}$ More detail about the calculation of $\chi_{e f f}^{(2)}$ can be seen from the Supplementary Note 3.

Polarization-resolved SHG measurements provide important crystallographic information of $\mathrm{WSe}_{2}$ atomic layers if the signal is collected through a linear polarized-analyzer set, due to the sensitivity of the SHG to crystal symmetry. To determine and distinguish the contributions of SHG signals influenced by the monolayer $\mathrm{WSe}_{2}$ crystal orientation and the trench orientation, a single crystalline monolayer $\mathrm{WSe}_{2}$ flake covering both gold nanostructure ( I ) and flat gold film (II) was measured (Figure 5a). The pump laser was polarized along the $y$ axis, and the parallel SHG signal was collected, i.e. also along the $y$ axis, where the sample was rotated clockwise by an angle of $\theta$ in steps of 5 degree. The trenches were initially aligned with the $x$ axis, and the offset angle between the armchair axis of the $\mathrm{WSe}_{2}$ flake and $y$ axis was $\theta_{0}$. The polar plots for SHG of $\mathrm{WSe}_{2}$ on gold trenches (Region I ) and on flat gold (Region II) are shown in Figure 5b and Figure S12a, respectively, where the SHG intensity is plotted as a function of the rotation angle $(\theta)$ of the sample. A typical petal pattern with six-fold symmetry is observed (Figure S12a and Supplementary Note 4) for $\mathrm{WSe}_{2}$ on flat gold where the SHG pattern is fitted with an equation:

$$
I_{2 \omega, W S e_{2}}^{\|} \text {on flat gold }=C_{1} \cos ^{2}\left[3\left(\theta+\theta_{0}\right)\right]
$$


where $C_{1}$ is a constant obtained from the largest SHG intensity. The peaks of SHG intensity point to the armchair directions in monolayer $\mathrm{WSe}_{2}$ lattice, i.e., the perpendicular bisectors of the single crystalline flake (blue dashed arrow). The fitting shown in red shows that the initial offset angle $\left(\theta_{0}\right)$ between the armchair direction of the single crystalline $\mathrm{WSe}_{2}$ and pump laser polarization is $10^{\circ}$, indicating that the armchair axis was initially rotated with respect to the pump laser. Unlike the polar pattern of Region II, Region I shows a polar pattern with two large and two small petals (Figure 5b) that can be fitted by the equation:

$$
I_{2 \omega, W S e_{2} \text { on trenches }}^{\|}=C_{2} \cos ^{2}\left[3\left(\theta+\theta_{0}\right)\right] \cdot \cos ^{4} \theta
$$

where $C_{2}$ is a constant that fits the SHG amplitude at each angle (see eq. S20). The first cosine term represents the influence of the crystal orientation and the second cosine term reflects how the angle between the trench and the $y$ axis influences the SHG intensity. The trenches were initially oriented perpendicular to the polarization of pump laser, in which case, the near field of the pump laser localized at the gap has the largest value: $\mathbf{E}_{\text {gap,initial }}^{\|}=f \mathbf{E}_{\omega}$, where $\mathbf{E}_{\text {gap }}^{\|}$is the enhanced local electric field with the same polarization direction as $\mathbf{E}_{\omega}$ and $f$ is the enhancement factor defined by $\mathbf{E}_{\text {gap }} / \mathbf{E}_{0}$. As the trenches rotate by angle $\theta$, the near field enhanced by the gap plasmon along the direction of pump laser is given by $\mathbf{E}_{\text {gap,rotated }}^{\|}=f \mathbf{E}_{\omega} \cos \theta$ (Figure S13). Therefore, the SHG intensity of the $\mathrm{WSe}_{2}$ on gold trenches varies with the rotation of the trench according to the $\cos ^{4} \theta$ function. More details can be seen in the Supplementary Note 5 and Figure S13.

The components of the second-order nonlinear susceptibility are provided in terms of the crystal axes $(a, b, c)$ as discussed in eq. 1. However, it is convenient to describe the nonlinear polarization in terms of the laboratory axes $(x, y, z)$ as the direction of propagation and 
polarizations of the interacting waves are defined relative to the laboratory axes. According to the transformation from the crystal coordinate to the laboratory coordinate, we can derive the effective second-order nonlinear susceptibility along $x$ and $y$ axes (see details in Figure S9 and the corresponding detailed description in Supporting Note 3). The parallel $\left(E_{2 \omega}^{\|}\right)$and perpendicular $\left(E_{2 \omega}^{\perp}\right)$ components of the second-harmonic electric field with respect to the pump laser polarization ( $y$ axis) can be expressed via the effective second-order nonlinear susceptibility: ${ }^{39}$

$$
\begin{aligned}
& \mathbf{E}_{2 \omega}^{\|} \propto\left|\chi_{b b b}^{(2)}\right| \cos 3\left(\theta+\theta_{0}\right) \\
& \mathbf{E}_{2 \omega}^{\perp} \propto\left|\chi_{b b b}^{(2)}\right| \sin 3\left(\theta+\theta_{0}\right)
\end{aligned}
$$

The fitting curves for the polarization-dependent SHG in Figure $5 b$ and Figure S12b are consistent with the theoretical verification of Equation 6. The total SHG intensity (without the analyzer) is expressed as follows:

$$
I_{2 \omega}=I_{2 \omega}^{\|}+I_{2 \omega}^{\perp}=C^{2}\left[\left(E_{2 \omega}^{\|}\right)^{2}+\left(E_{2 \omega}^{\perp}\right)^{2}\right] \propto C^{2}\left|\chi_{b b b}^{(2)}\right|^{2}
$$

where $I_{2 \omega}$ is constant with respect to the crystal orientation. Therefore, the crystal orientation influences the parallel or perpendicular component of SHG signal, but not the total SHG signal.

The power-dependent total $\mathrm{SH}$ signal from both $\mathrm{WSe}_{2}$ on gold trenches (Figure $5 \mathrm{c}$ ) and on flat gold film (Figure S12b) exhibit a quadratic input power dependence, and the fitting line (red) has a slope of $\sim 2$, in agreement with the second-order nature of the nonlinear process. As shown in Figure 5c, the experimental results (black squares) deviate from the fitting line (red) at higher excitation power and show a shallower slope with increasing pump power, indicating saturation in SHG. ${ }^{40-41}$

\section{CONCLUSION}


In summary, we present a hybrid nanostructure consisting of $\mathrm{WSe}_{2}$ on a gold film with trenches that support lateral gap plasmon resonances at $\sim 800 \mathrm{~nm}$. The hybrid nanostructure is flexible and significantly enhances second-harmonic emission at $400 \mathrm{~nm}$ by $\sim 7000$-fold, without emission peak broadening or increased spectral background at room temperature. As observed in both experiments and simulation, the augmented SHG is attributed to the enhancement of the local excitation fields within the sub-20-nm-wide trenches. Furthermore, the anisotropic gold substrate composed of trenches aligned along a single direction allows for dynamic tuning of the SHG amplitude from $\mathrm{WSe}_{2}$ by simply rotating the polarization direction of the pump laser. This hybrid nanostructure provides a convenient and practical approach to obtain blue emission from a near-infrared laser in monolayer TMDCs. Furthermore, it provides a proof-of-concept for ultrathin and flexible frequency converters with high monochromaticity, realizing a miniaturized bendable photonic device for various applications, e.g., information processing, and providing a new freedom of engineering the optical properties of $\mathrm{WSe}_{2}$ by strain imposed by the bendable substrate. $^{42}$

\section{METHODS}

Material growth and quality of $\mathbf{W S e}_{2}$. The monolayer $\mathrm{WSe}_{2}$ single crystal was grown on c-cut sapphire by a chemical vapor deposition (CVD) of $\mathrm{WO}_{3}$ and Se powders in a horizontal hot-wall chamber. The $\mathrm{WO}_{3}$ powders at the center heating zone were heated to $925{ }^{\circ} \mathrm{C}$, and the Se powders at the upper stream side were maintained at $270{ }^{\circ} \mathrm{C}$. The $\mathrm{WSe}_{2}$ samples were obtained on sapphire substrate placed at the downstream side using an $\mathrm{Ar} / \mathrm{H}_{2}$ flowing gas at 10 Torr for 15 min. The detailed growth procedures can be found elsewhere. ${ }^{43-44}$ This technique yields high quality monolayer $\mathrm{WSe}_{2}$ with a crystal size ranging from 4 to $15 \mu \mathrm{m}$ (Figure S1a). The typical 
PL spectrum of a pristine monolayer $\mathrm{WSe}_{2}$ (Figure S1e) is dominated by an emission peak at 765 $\mathrm{nm}(1.62 \mathrm{eV})$ that arises from the A direct excitonic transition. ${ }^{45-46}$ For monolayer $\mathrm{WSe}_{2}$ on sapphire, the Raman spectrum excited by a 532-nm laser shown in Figure S1f, has two characteristic peaks are at $250 \mathrm{~cm}^{-1}$ and $259 \mathrm{~cm}^{-1}$ that originate from the degenerate $\mathrm{E}^{\prime} / \mathrm{A}_{1}^{\prime}$ mode and 2LA(M) mode, respectively.

Fabrication of gold substrate and transferring of $\mathbf{W S e}_{2}$. The gold substrate consisting of gold trenches and flat gold film was prepared by a template-stripping method based on nano-patterned silicon templates. ${ }^{6,44}$ First, hydrogen silsesquioxane (HSQ) was spin coated onto a cleaned silicon substrate, and then was patterned by electron beam lithography (EBL). After development of the exposed HSQ, silicon etching and HSQ mask removal were carried out. Next, a 150-nmthick gold film was evaporated onto the silicon substrate, followed by a template-stripping process with a glass slide. More details can be seen in the supporting information. WSe $\mathrm{Wlake}_{2}$ was then transferred onto the gold substrate by a wet transfer approach. ${ }^{44,47}$ A layer of poly (methylmethacrylate) (PMMA) (950K A5) was first spin coated on the monolayer $\mathrm{WSe}_{2}$ flakes as a transfer supporting layer and then baked at $130{ }^{\circ} \mathrm{C}$ for $2 \mathrm{~min}$. The PMMA capped $\mathrm{WSe}_{2}$ was exfoliated from the sapphire substrate in a $\mathrm{NaOH}$ solution $(3 \mathrm{~mol} / \mathrm{L})$ at $100{ }^{\circ} \mathrm{C}$. After dilution of etchant and residues in deionized water, the PMMA-capped monolayer $\mathrm{WSe}_{2}$ was transferred onto the gold substrate, followed by the removal of PMMA film using acetone and sample cleaning by isopropyl alcohol. For making flexible devices, the gold substrate with $\mathrm{WSe}_{2}$ was peeled off from the glass slide by a tweezer and pasted onto a sticky bendable polydimethylsiloxane (PDMS) substrate (Figure S2).

Characterization. Otherwise stated specifically, the characterization was conducted on unbent samples. AFM images were obtained using a BRUKER Dimension FastScan equipment. The 
optical reflectance spectra of the samples were measured by using a CRAIC UV-VIS-NIR micro-spectrophotometer model QDI 2010 (equipped with a 36× objective lens with NA of 0.5). Moreover, SEM images were obtained by FEI Verios 460. A confocal microphotoluminescence/Raman setup was used to excite the sample using a 532-nm CW pump laser, which was focused by a $100 \times$ microscope objective lens. The SHG was measured by a femtosecond laser beam with a central wavelength of $800 \mathrm{~nm}, 825 \mathrm{~nm}, 850 \mathrm{~nm}, 875 \mathrm{~nm}$ or 900 $\mathrm{nm}$, a pulse duration of $140 \mathrm{fs}$ and a repetition rate of $80 \mathrm{MHz}$ at room temperature. The detailed setup was shown in Figure S3. There was no analyzer before the detectors except in the experiment of investigating the influence of crystal and trench orientation. Therefore, the total SHG intensity shown in Figure 1e-f, Figure 2c-d, Figure 3e-f, Figure 4a-b, Figure 5c, Figure S1, Figure S5, Figure S6, Figure S8, Figure S10, Figure S11, Figure S12b does not depend on the crystal orientation, while the parallel component of SHG intensity relative to the polarization of pump laser shown in Figure $5 \mathrm{~b}$ and Figure $\mathrm{S} 8 \mathrm{a}$ is related with the crystal orientation. We considered and experimentally calibrated the change of the S- and P-polarized light caused by optical components such as dichroic mirror and short pass, and the detecting sensibility of the spectrometer and PMT. For all SHG measurements, the pump laser power on the sample was typically set at $0.5 \mathrm{~mW}$, far below the saturation region, to prevent possible damage, with the exception of the experiment on power dependence in Figure 5c and Figure S12b. In addition, the SHG enhancement factor is defined by the ratio of the total SHG intensity from TMDCs on the trenches region to that from TMDCs on sapphire.

Numerical simulations. A commercial software package, Lumerical FDTD Solutions, was used
to simulate the electric field distributions. Periodic boundary conditions were used along the $x$ -
and $y$-axes, while a perfect-matched layer was used along $z$-axis. The finest mesh size was set to 
be $0.5 \mathrm{~nm}$ in the structure. The width of the metal nanostructures in the simulations was designed to match with the SEM images (image of Figure 2a, Figure 3a), with a trench depth of $34 \mathrm{~nm}$ for 910-nm pitch and $102 \mathrm{~nm}$ for 170-nm pitch. The depth of the trenches were estimated from the height of the ridge of the silicon template as shown in the AFM profile (Figure S14a-d).

\author{
ASSOCIATED CONTENT \\ AUTHOR INFORMATION \\ Corresponding Authors \\ *E-mail (J. K. W. Yang): joel_yang@sutd.edu.sg. \\ *E-mail (C. W. Qiu): chengwei.qiu@nus.edu.sg. \\ *E-mail (A. T. S. Wee): phyweets@nus.edu.sg
}

Author contributions

Z.W., Z.G.D., J.K.W.Y., C.W.Q and A.T.S.W. conceived the experiments; Z.W. transferred $\mathrm{WSe}_{2}$ on gold substrate, characterized the OM, AFM, SEM, Reflectance, Raman and PL. Z.G.D. and J. K. W. Y prepared the gold substrate. Z. W., H. Z. and Q. H. X. conducted the SHG measurement. L.J. and C.W.Q did the simulations, G.E. provided the PL/Raman system, M.H.C. and L.J.L. grew the $\mathrm{WSe}_{2}$ monolayers samples, S.A.M. provided expertise in data analysis. The paper was drafted by Z.W. with inputs from Z.G.D., J.K.W.Y., A.T.S.W., C.W.Q, S.A.M, G.E. and L.J. L. Z.W., Z.G.D. and H.Z contributed equally to this work.

The authors declare no competing financial interest.

\title{
ACKNOWLEDGEMENTS
}


Z. W. acknowledges scholarship support from NUS Graduate School for Integrative Sciences \& Engineering (NGS). Z.D. and J.K.W.Y. acknowledge the funding support from the Agency for Science, Technology and Research (A*STAR) Young Investigatorship (grant number 0926030138), SERC (grant number 092154099), the National Research Foundation (grant number NRF-CRP 8-2011-07), A*STAR Pharos Program (Grant No. 1527300025), and A*STAR-JCO under project number 1437C00135. C.-W.Q acknowledges the financial support from A*STAR Pharos Program (Grant No. 15270 00014, with Project No. R-263-000-B91-305). Z.W. and A.T.S.W. acknowledges the funding support from MOE Tier 2 grant R 144-000-382112 and facility support from NUS Center for Advanced 2D Materials. S.A.M. acknowledges the EPSRC Reactive Plasmonics Programme Grant (EP/M013812/1), the Royal Society, and the Lee-Lucas Chair in Physics. L.-J.L. acknowledges support from KAUST (Saudi Arabia) and Taiwan Consortium of Emergent Crystalline Materials (TCECM). G.E. acknowledges Singapore National Research Foundation, Prime Minister s Office, Singapore, for funding the research under its Medium-sized Centre program as well as NRF Research Fellowship (NRF-NRFF201102).

\section{Supporting information}

The Supporting Information is available free of charge via Internet at http://pubs.acs.org.

Characterization results of $\mathrm{WSe}_{2}$ on sapphire, flat gold and the gold trenches (Figure S1); Detailed illustration on the fabrication process of flexible hybrid structure (Figure S2); Optical setup for measuring SHG mappings and SHG spectra at room temperature (Figure S3); Photograph of $\mathrm{WSe}_{2}$ on gold substrate attached to an inward-bent polydimethylsiloxane (PDMS) substrate (Figure S4); SHG spectra of the sample ( $\mathrm{WSe}_{2}$ on nanostructure with a pitch of 170 $\mathrm{nm}$ ) before and after being bent 50 times (Figure S5); Gold trenches without WSe $\mathrm{W}_{2}$ has no SHG (Figure S6); Diagram showing the area of multiple trenches perpendicular to the polarization 
direction of the laser within a laser spot (Figure S7); Comparing spectra profile of second harmonic generation with central wavelength of $400 \mathrm{~nm}$ (SHG, red line, bottom axis) and fundamental wave (FW, black dot, top axis) with central wavelength of $800 \mathrm{~nm}$ (Figure S8); Demonstration of transformation from crystal coordinates $(a, b, c)$ to laboratory coordinates $(x, y$, z) (Figure S9); SHG intensity from monolayer $\mathrm{WSe}_{2}$ on trenches with a pitch of $170 \mathrm{~nm}$ and on sapphire as a function of pump wavelength ranging from $800 \mathrm{~nm}$ to $900 \mathrm{~nm}$ (Figure S10); Quantitative comparison of SHG from monolayer $\mathrm{WSe}_{2}, \mathrm{MoSe}_{2}$ and $\mathrm{MoS}_{2}$ on nanostructure with a pitch of $170 \mathrm{~nm}$ and on the as-grown sapphire (Figure S11); Dependence of the SHG on sample rotated angle, laser power and pump wavelength on SHG intensity, respectively (Figure $\mathrm{S} 12)$; Demonstration of the electric field amplitude as a function of the rotation angle $\theta$ of the trenches (Figure S13); Height of the ridges on silicon template (Figure S14) (PDF).

\section{REFERENCES}

(1) New, G. Frequency Mixing. In Introduction to Nonlinear Optics; Cambridge: Cambridge University Press, 2011; pp 19-44.

(2). Kumar, N.; Najmaei, S.; Cui, Q.; Ceballos, F.; Ajayan, P. M.; Lou, J.; Zhao, H. Second Harmonic Microscopy of Monolayer MoS 2 . Phys. Rev. B 2013, 87, 161403.

(3). Malard, L. M.; Alencar, T. V.; Barboza, A. P. M.; Mak, K. F.; de Paula, A. M. Observation of Intense Second Harmonic Generation from $\mathrm{MoS}_{2}$ Atomic Crystals. Phys. Rev. B 2013, 87, 201401.

(4). Kuo, P. S.; Bravo-Abad, J.; Solomon, G. S. Second-Harmonic Generation Using-QuasiPhasematching in a GaAs Whispering-Gallery-Mode Microcavity. Nat. Commun. 2014, 5, 3109. (5). Yu, H.; Talukdar, D.; Xu, W.; Khurgin, J. B.; Xiong, Q. Charge-Induced Second-Harmonic Generation in Bilayer WSe 2 . Nano Lett. 2015, 15, 5653-5657.

(6). Dong, Z.; Asbahi, M.; Lin, J.; Zhu, D.; Wang, Y. M.; Hippalgaonkar, K.; Chu, H.-S.; Goh, W. P.; Wang, F.; Huang, Z.; Yang, J. K. W. Second-Harmonic Generation from Sub-5 nm Gaps by Directed Self-Assembly of Nanoparticles onto Template-Stripped Gold Substrates. Nano Lett. 2015, 15, 5976-5981.

(7). Zheludev, N. I.; Kivshar, Y. S. From Metamaterials to Metadevices. Nat. Mater. 2012, 11, 917. 
(8). Wang, C.-Y.; Guo, G.-Y. Nonlinear Optical Properties of Transition-Metal Dichalcogenide $\mathrm{MX}_{2}(\mathrm{M}=\mathrm{Mo}, \mathrm{W} ; \mathrm{X}=\mathrm{S}, \mathrm{Se})$ Monolayers and Trilayers from First-Principles Calculations. $J$. Phys. Chem. C 2015, 119, 13268-13276.

(9). Ribeiro-Soares, J.; Janisch, C.; Liu, Z.; Elías, A.; Dresselhaus, M.; Terrones, M.; Cançado, L.; Jorio, A. Second Harmonic Generation in $\mathrm{WSe}_{2}$. 2D Mater. 2015, 2, 045015. (10). Shoji, I.; Kondo, T.; Kitamoto, A.; Shirane, M.; Ito, R. Absolute Scale of Second-Order Nonlinear-Optical Coefficients. J. Opt. Soc. Am. B 1997, 14, 2268-2294.

(11). Janisch, C.; Wang, Y.; Ma, D.; Mehta, N.; Elías, A. L.; Perea-López, N.; Terrones, M.; Crespi, V.; Liu, Z. Extraordinary Second Harmonic Generation in Tungsten Disulfide Monolayers. Sci. Rep. 2014, 4, 5530.

(12). Fryett, T.; Zhan, A.; Majumdar, A. Cavity Nonlinear Optics with Layered Materials. Nanophotonics 2018, 7, 355-370.

(13). Clark, D.; Senthilkumar, V.; Le, C.; Weerawarne, D.; Shim, B.; Jang, J.; Shim, J.; Cho, J.; Sim, Y.; Seong, M.-J. Strong Optical Nonlinearity of CVD-Grown $\mathrm{MoS}_{2}$ Monolayer as Probed by Wavelength-Dependent Second-Harmonic Generation. Phys. Rev. B 2014, 90, 121409. (14). Zhao, M.; Ye, Z.; Suzuki, R.; Ye, Y.; Zhu, H.; Xiao, J.; Wang, Y.; Iwasa, Y.; Zhang, X. Atomically Phase-Matched Second-Harmonic Generation in a 2D Crystal. Light Sci. Appl. 2016, 5 , e16131.

(15). Klein, J.; Wierzbowski, J.; Steinhoff, A.; Florian, M.; Rösner, M.; Heimbach, F.; Müller, K.; Jahnke, F.; Wehling, T. O.; Finley, J. J. Electric-Field Switchable Second-Harmonic Generation in Bilayer $\mathrm{MoS}_{2}$ by Inversion Symmetry Breaking. Nano Lett. 2016, 392-398.

(16). Li, Y.; Rao, Y.; Mak, K. F.; You, Y.; Wang, S.; Dean, C. R.; Heinz, T. F. Probing Symmetry Properties of Few-Layer $\mathrm{MoS}_{2}$ and h-BN by Optical Second-Harmonic Generation. Nano Lett. 2013, 13, 3329-3333.

(17). Chen, H.; Corboliou, V.; Solntsev, A. S.; Choi, D.-Y.; Vincenti, M. A.; de Ceglia, D.; De Angelis, C.; Lu, Y.; Neshev, D. N. Enhanced Second-Harmonic Generation from TwoDimensional $\mathrm{MoSe}_{2}$ on a Silicon Waveguide. 2017, (6). (18). Wang, G.; Marie, X.; Gerber, I.; Amand, T.; Lagarde, D.; Bouet, L.; Vidal, M.; Balocchi, A.; Urbaszek, B. Giant Enhancement of the Optical Second-Harmonic Emission of $\mathrm{WSe}_{2}$ Monolayers by Laser Excitation at Exciton Resonances. Phys. Rev. Lett. 2015, 114, 097403. (19). Seyler, K. L.; Schaibley, J. R.; Gong, P.; Rivera, P.; Jones, A. M.; Wu, S.; Yan, J.; Mandrus, D. G.; Yao, W.; Xu, X. Electrical control of second-harmonic generation in a $\mathrm{WSe}_{2}$ monolayer transistor. Nat. Nanotechnol. 2015, 10 (5), 407-411.

(20). Rodrigues, S. P.; Cai, W. Nonlinear Optics: Tuning Harmonics with Excitons. Nat. Nanotechnol. 2015, 10, 387-388.

(21). Yin, X.; Ye, Z.; Chenet, D. A.; Ye, Y.; O’Brien, K.; Hone, J. C.; Zhang, X. Edge Nonlinear Optics on a $\mathrm{MoS}_{2}$ Atomic Monolayer. Science 2014, 344, 488-490.

(22). Fryett, T. K.; Seyler, K. L.; Zheng, J.; Liu, C.-H.; Xu, X.; Majumdar, A. Silicon Photonic Crystal Cavity Enhanced Second-Harmonic Generation from Monolayer WSe 2 . 2D Mater. 2016, $4(1), 015031$.

(23). Day, J. K.; Chung, M.-H.; Lee, Y.-H.; Menon, V. M. Microcavity Enhanced Second Harmonic Generation in 2D MoS 2 . Opt. Mater. Express 2016, 6, 2360-2365.

(24). Yi, F.; Ren, M.; Reed, J. C.; Zhu, H.; Hou, J.; Naylor, C. H.; Johnson, A. C.; Agarwal, R.; Cubukcu, E. Optomechanical Enhancement of Doubly Resonant 2D Optical Nonlinearity. Nano Lett. 2016, 16 (3), 1631-1636. 
(25). Wu, S.; Buckley, S.; Schaibley, J. R.; Feng, L.; Yan, J.; Mandrus, D. G.; Hatami, F.; Yao, W.; Vučković, J.; Majumdar, A. Monolayer Semiconductor Nanocavity Lasers with Ultralow Thresholds. Nature 2015, 520, 69-72.

(26). Kildishev, A. V.; Boltasseva, A.; Shalaev, V. M. Planar Photonics with Metasurfaces. Science 2013, 339, 1232009.

(27). Avayu, O.; Almeida, E.; Prior, Y.; Ellenbogen, T. Composite Functional Metasurfaces for Multispectral Ahromatic Optics. Nat. Commun. 2017, 8, 14992.

(28). Keren-Zur, S.; Avayu, O.; Michaeli, L.; Ellenbogen, T. Nonlinear Beam Shaping with Plasmonic Metasurfaces. ACS Photonics 2015, 3, 117-123.

(29). Zharov, A. A.; Shadrivov, I. V.; Kivshar, Y. S. Nonlinear Properties of Left-Handed Metamaterials. Phys. Rev. Lett. 2003, 91, 037401.

(30). Wang, Q. H.; Kalantar-Zadeh, K.; Kis, A.; Coleman, J. N.; Strano, M. S. Electronics and Optoelectronics of Two-Dimensional Transition Metal Dichalcogenides. Nat. Nanotechnol. 2012, 7, 699-712.

(31). Kern, J.; Trügler, A.; Niehues, I.; Ewering, J.; Schmidt, R.; Schneider, R.; Najmaei, S.; George, A.; Zhang, J.; Lou, J. Nanoantenna-Enhanced Light-Matter Interaction in Atomically Thin $\mathrm{WS}_{2}$. ACS Photonics 2015, 2, 1260-1265.

(32) Maier, S. A. Electromagnetics of Metals. In Plasmonics: Fundamentals and Applications; Springer Science \& Business Media: New York, 2007; pp 17-19.

(33). Wang, F. X.; Rodríguez, F. J.; Albers, W. M.; Ahorinta, R.; Sipe, J.; Kauranen, M. Surface and Bulk Contributions to the Second-Order Nonlinear Optical Response of a Gold Film. Phys. Rev. B 2009, 80, 233402.Co

(34). Wang, G.; Glazov, M.; Robert, C.; Amand, T.; Marie, X.; Urbaszek, B. Double Resonant Raman Scattering and Valley Coherence Generation in Monolayer WSe $\mathrm{W}_{2}$. Phys. Rev. Lett. 2015, 115 (11), 117401.

(35). Jiang, T.; Liu, H.; Huang, D.; Zhang, S.; Li, Y.; Gong, X.; Shen, Y.-R.; Liu, W.-T.; Wu, S. Valley and Band Structure Engineering of Folded $\mathrm{MoS}_{2}$ Bilayers. Nat. Nanotechnol. 2014, 9 , 825-829.

(36). Aouani, H.; Navarro-Cia, M.; Rahmani, M.; Sidiropoulos, T. P.; Hong, M.; Oulton, R. F.; Maier, S. A. Multiresonant Broadband Optical Antennas as Efficient Tunable Nanosources of Second Harmonic Light. Nano Lett. 2012, 12, 4997-5002.

(37). Gomez-Diaz, J. S.; Tymchenko, M.; Lee, J.; Belkin, M. A.; Alù, A. Nonlinear Processes in Multi-Quantum-Well Plasmonic Metasurfaces: Electromagnetic Response, Saturation Effects, Limits, and Potentials. Phys. Rev. B 2015, 92, 125429.

(38). Lee, J.; Tymchenko, M.; Argyropoulos, C.; Chen, P.-Y.; Lu, F.; Demmerle, F.; Boehm, G.; Amann, M.-C.; Alu, A.; Belkin, M. A. Giant Nonlinear Response from Plasmonic Metasurfaces Coupled to Intersubband Transitions. Nature 2014, 511, 65-69.

(39). Ko, T. Y.; Jeong, A.; Kim, W.; Lee, J.; Kim, Y.; Lee, J. E.; Ryu, G. H.; Park, K.; Kim, D.; Lee, Z. On-Stack Two-Dimensional Conversion of $\mathrm{MoS}_{2}$ into $\mathrm{MoO}_{3}$. 2 D Mater. 2016, 4, 014003. (40). Leo, F.; Hansson, T.; Ricciardi, I.; De Rosa, M.; Coen, S.; Wabnitz, S.; Erkintalo, M. Walk-off-Induced Modulation Instability, Temporal Pattern Formation, and Frequency Comb Generation in Cavity-Enhanced Second-Harmonic Generation. Phys. Rev. Lett. 2016, 116, 033901.

(41). Buckley, S.; Radulaski, M.; Biermann, K.; Vučković, J. Second Harmonic Generation in Photonic Crystal Cavities in (111)-Oriented GaAs. Appl. Phys. Lett. 2013, 103, 21111. 
(42). Li, J.; Shan, Z.; Ma, E. Elastic Strain Engineering for Unprecedented Materials Properties. MRS Bulletin 2014, 39, 108-114.

(43). Huang, J.-K.; Pu, J.; Hsu, C.-L.; Chiu, M.-H.; Juang, Z.-Y.; Chang, Y.-H.; Chang, W.-H.; Iwasa, Y.; Takenobu, T.; Li, L.-J. Large-Area Synthesis of Highly Crystalline $\mathrm{WSe}_{2}$ Monolayers and Device Applications. ACS Nano 2014, 8, 923-930.

(44). Wang, Z.; Dong, Z.; Gu, Y.; Chang, Y.-H.; Zhang, L.; Li, L.-J.; Zhao, W.; Eda, G.; Zhang, W.; Grinblat, G. Giant Photoluminescence Enhancement in Tungsten-Diselenide-Gold Plasmonic Hybrid Structures. Nat. Commun. 2016, 7, 11283.

(45). Zheng, Y. J.; Huang, Y. L.; Chen, Y.; Zhao, W.; Eda, G.; Spataru, C. D.; Zhang, W.; Chang, Y.-H.; Li, L.-J.; Chi, D. Heterointerface Screening Effects Between Organic Monolayers and Monolayer Transition Metal Dichalcogenides. ACS Nano 2016, 10, 2476-2484. (46). Jones, A. M.; Yu, H.; Ghimire, N. J.; Wu, S.; Aivazian, G.; Ross, J. S.; Zhao, B.; Yan, J.; Mandrus, D. G.; Xiao, D. Optical Generation of Excitonic Valley Coherence in Monolayer WSe $\mathrm{Se}_{2}$ Nat. Nanotechnol. 2013, 8, 634-638.

(47). Song, Z.; Schultz, T.; Ding, Z.; Lei, B.; Han, C.; Amsalem, P.; Lin, T.; Chi, D.; Wong, S. L.; Zheng, Y. J. Electronic Properties of a 1D Intrinsic/p-Doped Heterojunction in a 2D Transition Metal Dichalcogenide Semiconductor. ACS Nano 2017, 11, 9128-9135. 
a

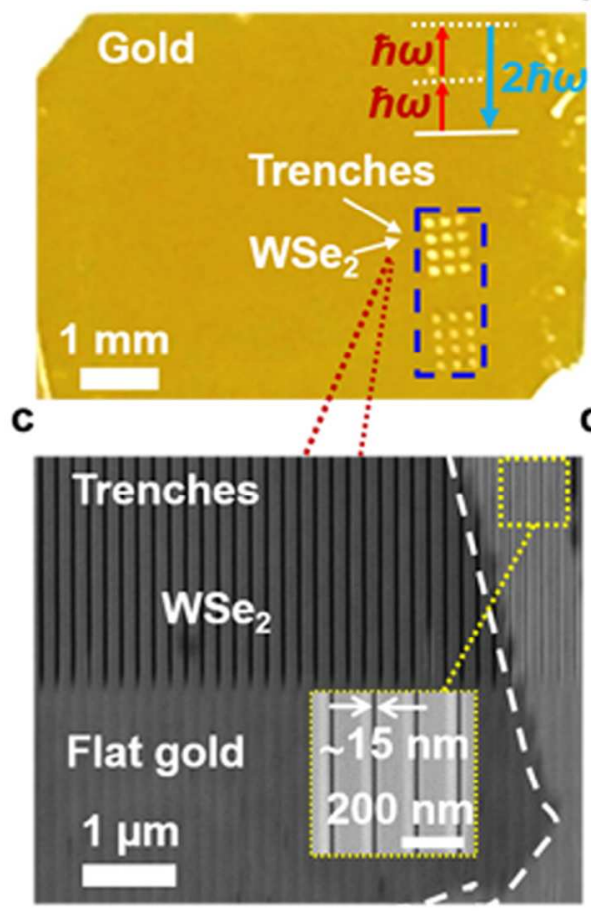

e $\times 10^{4}$ b
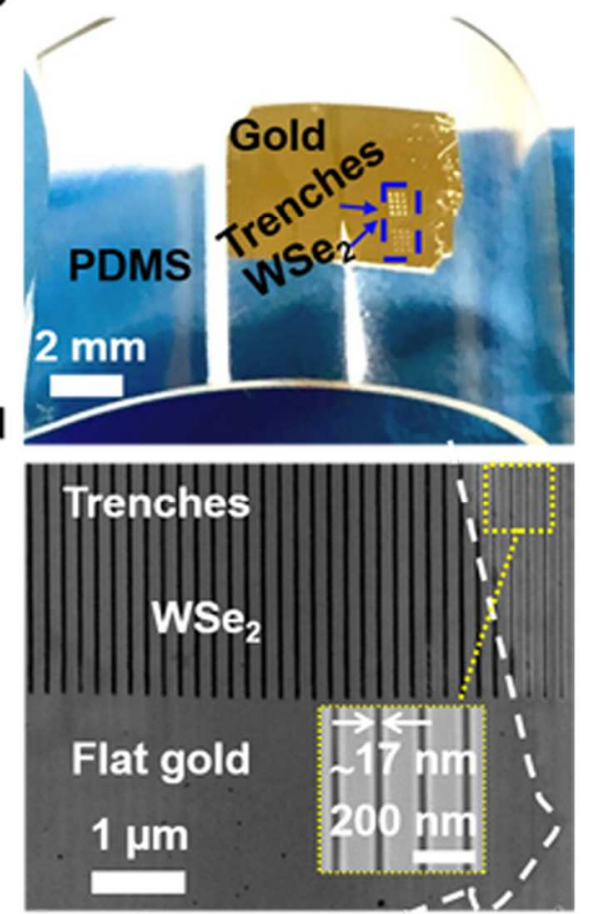

$\mathrm{f} \times 10^{4}$
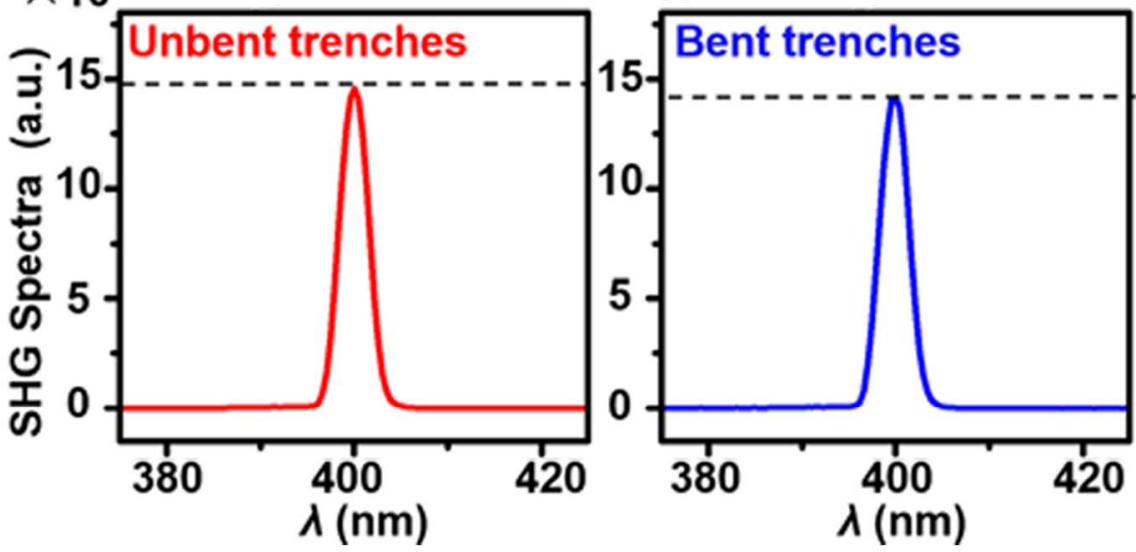

Figure 1. Efficient second-harmonic generation (SHG) from ultrathin and flexible hybrid nanostructure of monolayer WSe2 on gold trenches. (a,b) Photographs of WSe2 on gold substrate consisting of sub-20-nmwide trenches. The sample was attached to a flat unbent (a) and bent (b) polydimethylsiloxane (PDMS) substrate, respectively. Monolayer WSe2 was transferred onto a region with trenches and flat gold film as outlined in blue dashed lines. (c,d) SEM images of the unbent (c) and outward-bent (d) hybrid nanostructure of WSe2 on gold substrate, respectively. (e,f) SHG spectra of the unbent (e) and outward-bent (f) hybrid nanostructure as conducted by $800-\mathrm{nm}$ fs pump laser. The white dashed lines outline the region with monolayer WSe2. The yellow dotted line denotes a trench region without WSe2 enlarged in the inset. The trenches were spaced at a pitch of $170 \mathrm{~nm}$. 


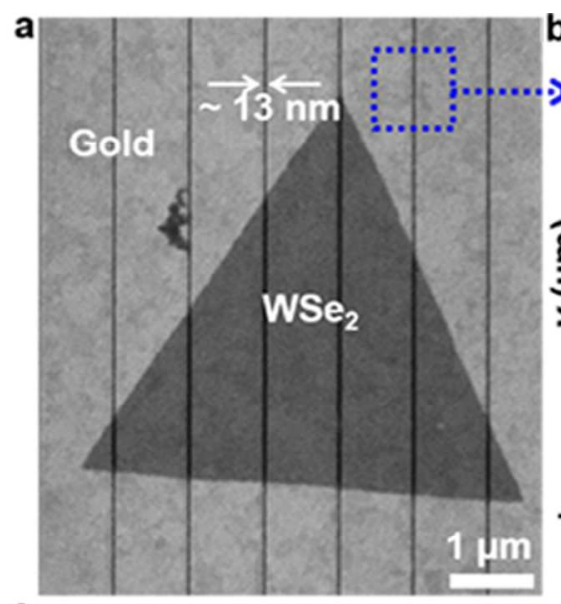

b1.8

c
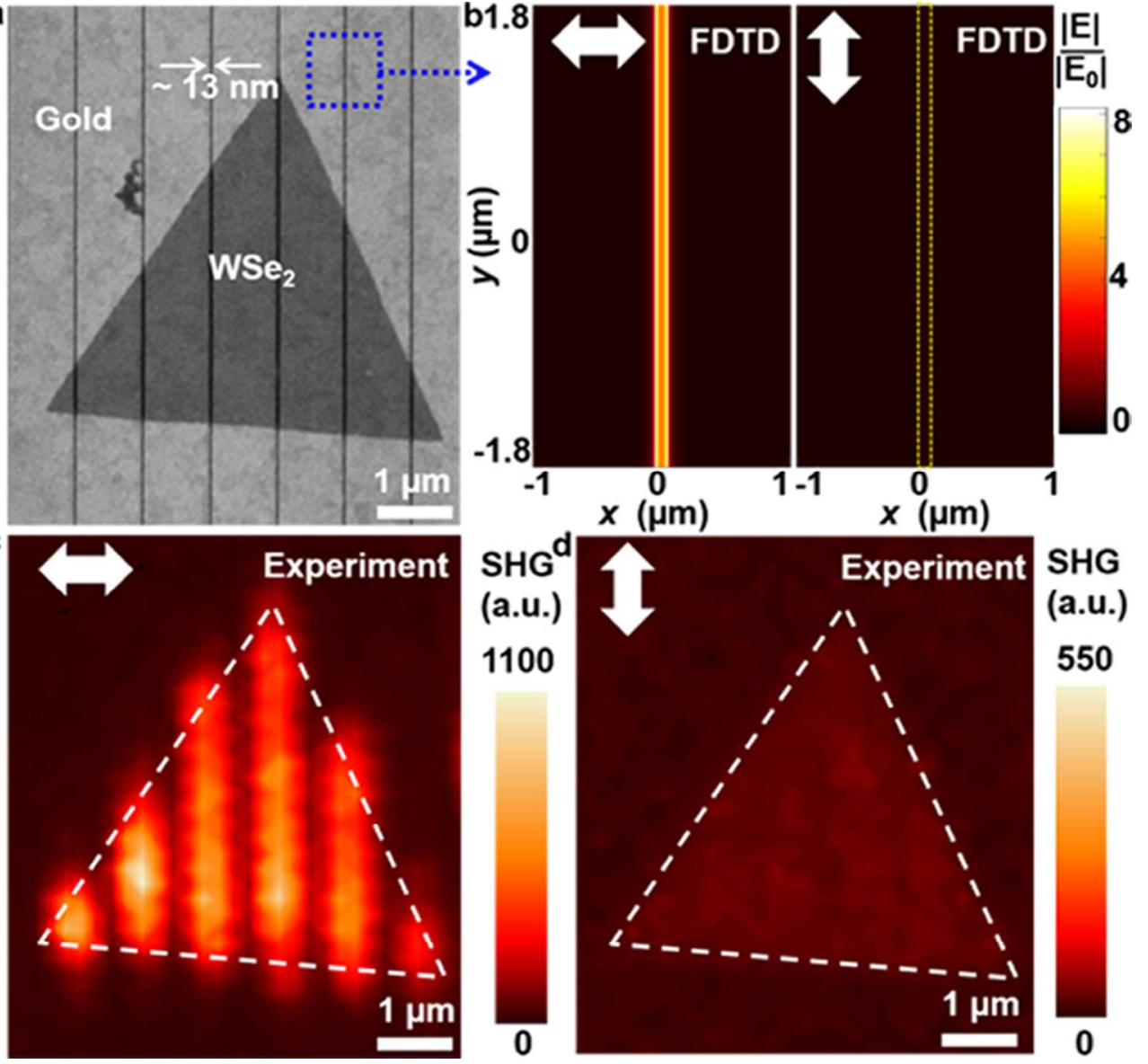

Experiment

SHG
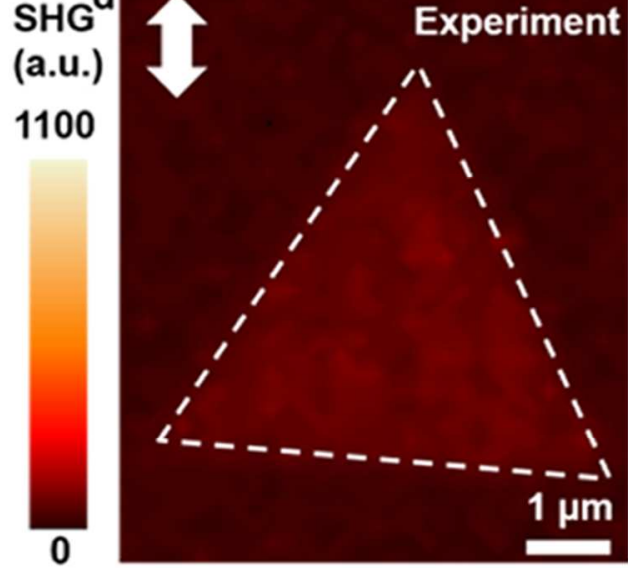

(a.u.)

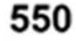

Figure 2. Pump-laser-polarization dependent SHG mapping. (a) SEM image of single-crystalline monolayer WSe2 flake on trenches with a pitch of $910 \mathrm{~nm}$. (b) Simulated electric field distribution at a plane $1 \mathrm{~nm}$ above the surface of gold substrate with pump laser polarized perpendicular (left panel) and parallel (right panel) to the trench. The dotted line outlines the geometry of the trench. (c,d) Corresponding experimental SHG mappings of the exact WSe2 flake on trenches as shown in the SEM image in (a) under resonant and non-resonant excitations, respectively. White dashed lines outline the WSe2 flake. The white arrows show the polarization directions of the pump laser.

$150 \times 135 \mathrm{~mm}(150 \times 150 \mathrm{DPI})$ 


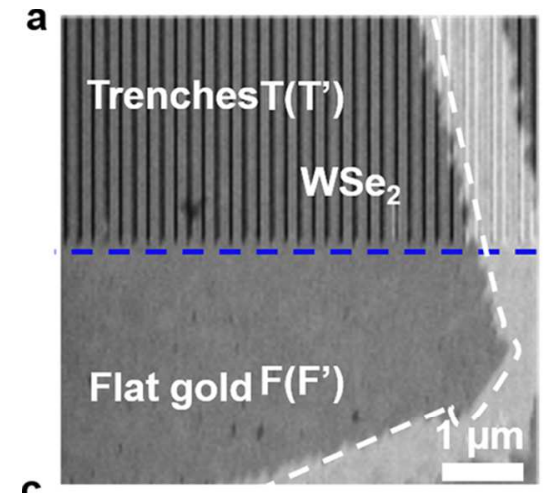

c
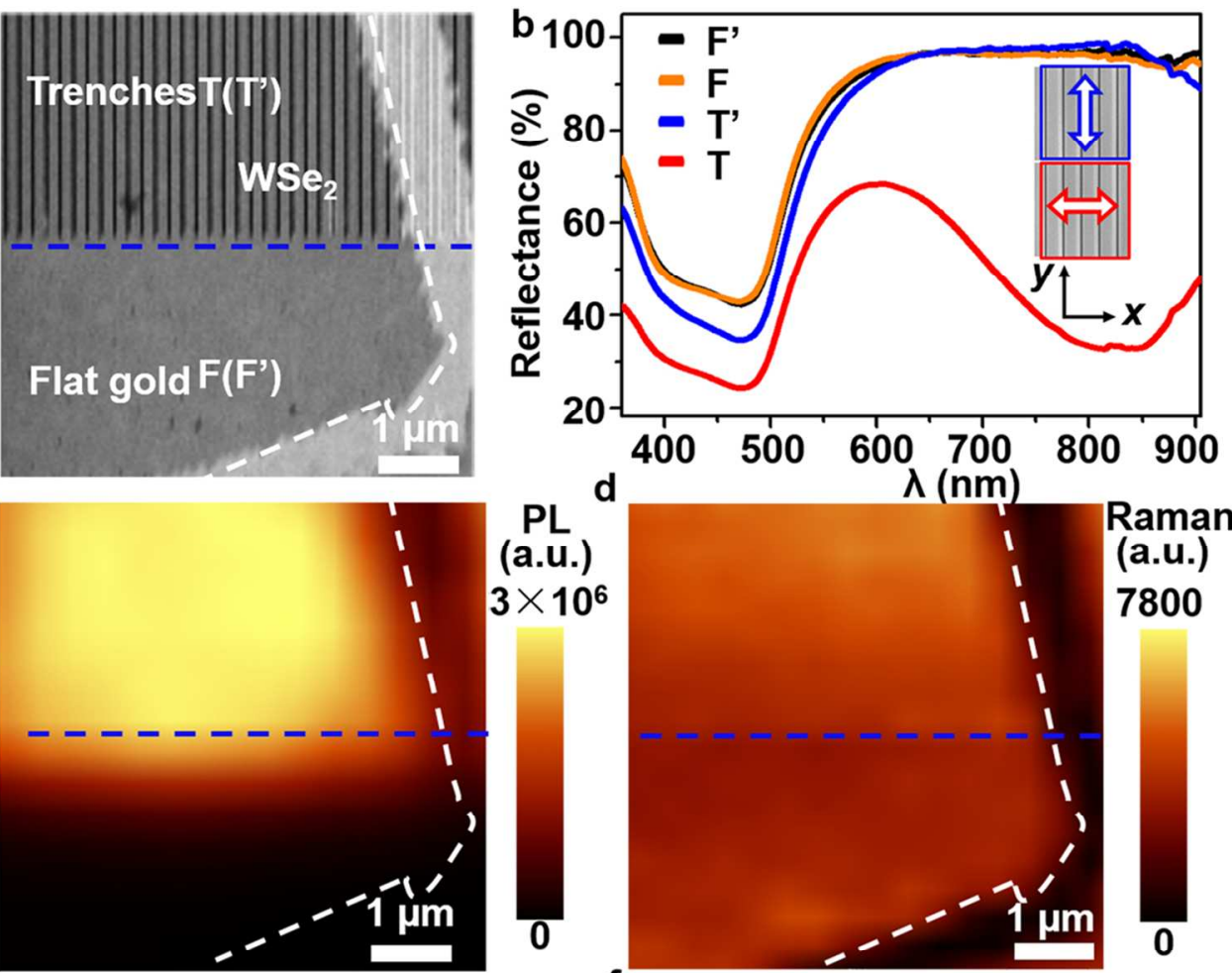

d
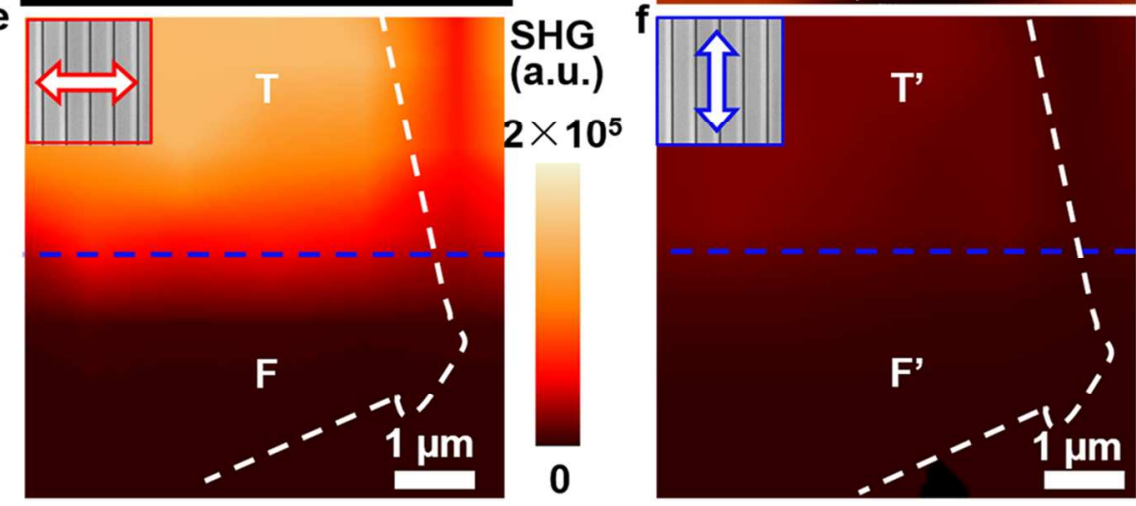

SHG

(a.u.)

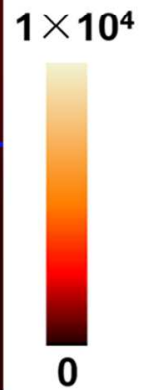

Figure 3. Maximum SHG enhancement from WSe2 on trenches with plasmon resonance aligned with 800-nm pump laser. (a) SEM image of a monolayer WSe2 flake on gold trenches with pitch of $170 \mathrm{~nm}$ (dark grey) denoted as $T\left(T^{\prime}\right)$ and flat gold film (grey) denoted as $F\left(F^{\prime}\right)$. White dashed line outlines the edges of WSe2. The blue dashed line outlines the boundary of trenches and flat gold film. (b) Reflectance spectra of gold trenches $\left(T\right.$ or $\left.\mathrm{T}^{\prime}\right)$ and flat gold film ( $\mathrm{F}$ or $\mathrm{F}^{\prime}$ ) under the illumination of white light polarized perpendicular $(\mathrm{x})$ or parallel $(y)$ to the trenches orientation. The gold trenches have lateral gap plasmon resonance at $\sim 800$ $\mathrm{nm}$ under the illumination of x-polarized light. (c,d) PL (c) and Raman (d) intensity mappings of WSe2 flakes on gold trenches and flat gold film. The intensity value at each pixel for PL mapping was obtained by integrating the PL spectrum across the spectrum window of $700-820 \mathrm{~nm}$, for Raman is the spectrum window of $245 \mathrm{~cm}-1-265 \mathrm{~cm}-1$. (e,f) SHG peak intensity mapping of WSe2 on gold trenches $\left(T\right.$ and $\left.T^{\prime}\right)$ and flat gold film ( $\mathrm{F}$ and $\mathrm{F}^{\prime}$ ) under resonant and non-resonant excitation, respectively. The pump laser is $800 \mathrm{~nm}$ with a power of $0.5 \mathrm{mw}$. 
b

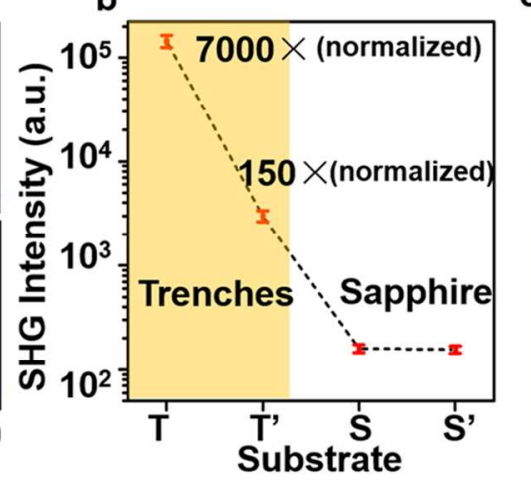

c

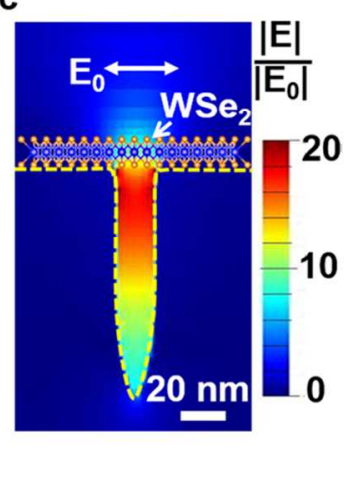

Figure 4. Quantitative comparison of SHG intensity. (a) Representative SHG spectra from WSe2 on gold trenches ( $\left(T\right.$ or $T^{\prime}$ ) and sapphire ( $S$ or $\left.S^{\prime}\right)$ measured by using $x$ - or $y$-polarized pump laser, respectively. (b) SHG peak intensity with an error bar of WSe2 on gold trenches and on sapphire. Ten data points were directly collected from the SHG mapping for each position without normalization of the trench area. The '7000' and '150' denote the normalized SHG enhancement factor under resonant and non-resonant excitations, respectively, where WSe2 on sapphire was used as reference. (c) Finite-difference time domain (FDTD) simulation of the electric field distribution of the lateral gap plasmons with a WSe2 monolayer flake being suspended over a single trench with a maximum width of $\sim 18 \mathrm{~nm}$ and a pitch of $170 \mathrm{~nm}$. The polarization of the incident laser field is across the gap. The dashed yellow line denotes the boundary between air and gold.

$99 \times 38 \mathrm{~mm}(300 \times 300$ DPI $)$ 


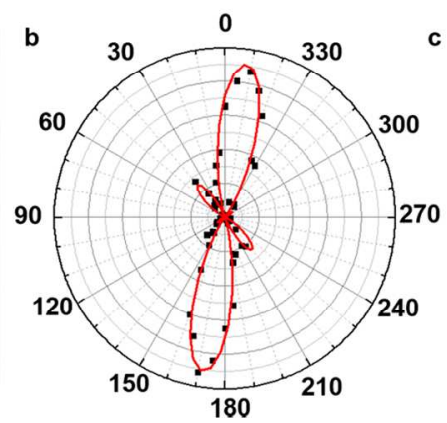

c

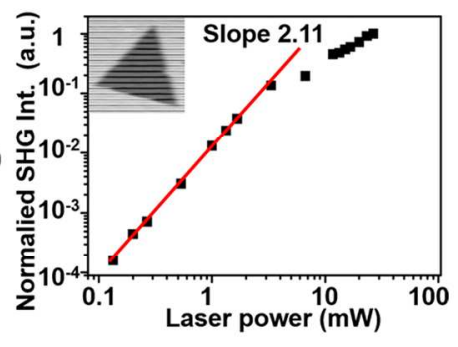

Figure 5. Dependence of the SHG on sample-rotation angle, laser power and pump wavelength on SHG intensity, respectively. (a) SEM image of WSe2 flake on gold trenches with pitch of $170 \mathrm{~nm}$ ( I ) and on flat gold film ( I ). White dashed line outlines the region of the investigated WSe2 flake with blue dashed arrows denoting the armchair directions. In the crystal coordinate, $b$ axis denotes one of the armchair direction and a axis denotes the zigzag direction. In the laboratory coordinate, $y$ axis denotes the polarization direction of $\mathrm{E} \omega . \theta$ is the rotate angle of the sample. $\Theta 0$ is the initial angle between the armchair direction of the WSe2 flake and $E \omega$. We only consider the parallel component of SHG signal relative to the polarization of the pump laser. The yellow bar denotes the orientation of the trench. (b) Polar plot of the normalized parallel component of SHG intensity from Region I for the entire $360^{\circ}$ rotation of the sample. The black squares are experimental data with fits shown in red according to the symmetry analysis in the text. (c) Normalized SHG intensity from Region I as a function of laser power in log-log scale plot, respectively.

$198 \times 71 \mathrm{~mm}(300 \times 300 \mathrm{DPI})$ 